\title{
Digitally Interpreting Traditional Folk Crafts
}

\author{
T. Vilbrandt ${ }^{1}$, C. Vilbrandt ${ }^{2}$, G. Pasko ${ }^{3}$, C. Stamm ${ }^{4}$, A. Pasko ${ }^{5}$ \\ ${ }^{1}$ Digital Materialization Lab, Japan, turlif@turlif.org \\ 2 Digital Materialization Lab, Chile, carl@cgpl.org \\ ${ }^{3}$ British Institute of Technology and E-commerce, gip@pasko.org \\ ${ }^{4}$ Uformia, Norway, cherie@uformia.no \\ ${ }^{5}$ Bournemouth University, UK, apasko@bournemouth.ac.uk
}

\begin{abstract}
The cultural heritage preservation requires that objects persist throughout time to continue to communicate an intended meaning. The necessity of computer-based preservation and interpretation of traditional folk crafts is validated by the decreasing number of masters, fading technologies, and crafts losing economic ground. We present a long-term applied research project on the development of a mathematical basis, software tools, and technology for application of desktop or personal fabrication using compact, cheap, and environmentally friendly fabrication devices, including '3D printers', in traditional crafts. We illustrate the properties of this new modeling and fabrication system using several case studies involving the digital capture of traditional objects and craft patterns, which we also reuse in modern designs. The test application areas for the development are traditional crafts from different cultural backgrounds, namely Japanese lacquer ware and Norwegian carvings. Our project includes modeling existing artifacts, Web presentations of the models, automation of the models fabrication, and the experimental manufacturing of new designs and forms.
\end{abstract}

Keywords: cultural heritage interpretation, craft, lacquer ware, carving, desktop fabrication

\section{INTRODUCTION}

The digital preservation and interpretation of cultural heritage has attracted considerable attention in computer graphics, geometric modeling, virtual reality and general computer science communities [1, 2]. One of the important characteristics of the object in digital preservation is its geometric shape, especially for threedimensional physical artifacts in various crafts, sculpture and architecture.

Any traditional craft is a living tradition, not a fixed set of inherited items. It includes masters with their knowledge of the essential craft technology, which is often not presented in written form. Traditional crafts preserve the shapes by passing them through successive generations of masters in the process of continuous reproduction of the craft items. As the number of masters decreases, technologies fade, and crafts lose economic ground, heritage preservation offers a chance for these traditional crafts to be captured and preserved in time. Yet it is possible to evolve some of these traditions by melding them with modern digital technologies allowing not only the preservation of these skills and objects, but the opportunity for these crafts to continue to be practised. One such technology, digital fabrication, continues to gain attention moving beyond its traditional uses for prototyping and industrial manufacturing. Where cultural heritage and digital fabrication meet is possible to preserve the living traditions of crafts, while offering new approaches to their design and production.

In this paper, we present a long-term applied research project on the development of a mathematical basis, software tools combining both interactive and automatic design pattern generation; and technology for application of modern digital fabrication using compact, cheap, and environmentally friendly devices in the area of traditional crafts. We illustrate the properties of this new modeling and fabrication system using two case 
studies from different cultural backgrounds, Japan and Norway, involving the digital capture of traditional objects and craft patterns, which we also reuse in modern designs. The first case study illustrates interactive modeling related to traditional crafts while another demonstrates an application of the automatic model generation. The last section provides the overall system evaluation and discussion.

\section{RELATED WORKS}

The areas of cultural heritage dealing with geometric shapes of physical artifacts include table ware (see practical examples of computer-aided design techniques related to this craft in [3]), pottery [4], textiles and embroidery [5], sculpture [6], and architecture [7]. Our work is oriented towards traditional crafts related to various types of sculpting and carving. Two categories of related works in this area can be distinguished: works dealing with interactive design operations and works investigating methods of automation of the models construction.

Besides the works on general computer-aided interactive sculpting techniques [8] and existing general purpose industrial surface sculpting tools such as ZBrush [9], some applied research results related to interactive sculpting and carving oriented to crafts have been published. Mizuno et al. [10] presented an approach to interactive virtual sculpting imitating the traditional technique of applying an elliptical chisel to carve the initial surface. Examples of created sculptures and woodcut printing blocks were presented. The underlying model for the presented system was Constructive Solid Geometry (CSG) based on set-theoretic operations applied to a limited set of primitives. The system was further extended by adding interactive operations with pressuresensitive pen thus imitating the work with real chisels on a wooden workpiece [11]. Interactive re-creation of traditional designs as well as creation of new designs using an interactive embossing technique was presented in [12]. Later in [13] the system was extended to support real-time interactive modeling operations such as sculpting, embossing, engraving, carving, and 3D painting.

In addition to interactive sculpting, some techniques for the automation of sculpting and carving operations were proposed. The automatic generation of woodblock models from a given image was described in [14]. A CSG model of a virtual wood block is generated by isolating carving areas in the greyscale image followed by analysis of features of the area and selecting an appropriate carving primitive for the area. The system was applied to reconstruct the printing woodblocks for traditional Japanese multicolor prints called ukiyo-e. Different techniques of depth data generation for automatic relief carving were described in [15]: polygon-to-function conversion, pattern dependent interpolation and ray-casting 3D models; while the carving operation itself was implemented using offsetting with the offset distance modulated by the generated depth data. Another approach to the automation of the model generation is based on fitting an existing parameterized template model to a point cloud data of a scanned artifact instance [16]. This approach was extended in [17] by the automatic generation of the template construction tree using the segmented point cloud.

It is worth to note that the most of above works employ constructive shape representations such as CSG and the Function Representation (FRep) [18, 19]. These representations allow for creation of 3D models that are as complete as possible and represent objects' internal structures, design logic (showing how simple components are interconnected into complex structures), and history of the shape construction, as well as time-dependent 
aspects and other parametric dependencies. More detailed discussions of shape representations for the cultural heritage preservation can be found in the next section and in [16].

Experiments have been reported on scanning, reconstruction and rapid prototyping (3D printing) of cultural heritage objects $[20,21,22]$. Such physical replicas can replace original artifacts at exhibitions and allow visitors to physically interact with artifacts. Large scale, expensive, not environmentally friendly industrial equipment that had no relation to the traditional materiality of the original artifacts was typically used in these experiments. Our interest is to introduce low-cost, non-toxic desktop fabrication devices into the processes of traditional crafts, helping to keep the craft alive by both using more traditional materials and providing a gateway for a new generation craftsmen.

\section{PROJECT OVERVIEW AND SHAPE REPRESENTATION ISSUES}

The purposes of our project are reflected in the following directions of research and development activity:

- Modeling shapes and making parametric families of models of representative craft items - a parametric family of models will allow us to generate samples of a specific model with different size, width/height ratio, and so on, without repetition of the entire modeling process

- $\quad$ Producing 3D virtual craft objects and presenting them on the Web

- Documenting traditional materials and technology. This documentation can also be presented in multimedia format including video, graphics, and virtual models

- Development of interactive design tools for modeling new items. This is a radical step of developing special computer-aided design (CAD) tools for modeling shapes and material properties of a particular craft

- Applying existing digital fabrication machines to produce 3D physical objects from computer models

- Adaptation or design of new digital fabrication tools for desktop manufacturing of craft items

- Creation of an Internet-based community and e-commerce activity using interactive computer-aided design, virtual objects presentation, and fabrication of selected or designed items by user request.

The implementation of the listed directions greatly depends on the selected mathematical representation of shapes. There are different approaches to digital representation of shapes of culturally valuable objects. Currently dominating Boundary Representation (BRep) in the form of polygonal meshes and parametric surfaces serve well the purposes of interactive visualization. As soon as the purpose of the application is looking to understand the physical structure and techniques related to an artifact, representing parametric families of artifacts, Web based modeling, long-term archiving (hundreds of years) or physical reproduction through digital fabrication, one encounters numerous quantitative (model size and processing time) and qualitative problems (model validity, precision, parameterization, operability, and manufacturability) of BRep:

Size and processing time 
Surface based models of a moderate size containing high quality details can include such a huge number of polygons that it becomes difficult or impossible for modern graphics hardware to render. Problems of file sizes become even more serious when Web-based presentation and manipulation of models is involved.

\section{Validity and precision}

The known problems arising from traditional BRep based CAD models (such as cracks in surfaces, selfintersections of polygons, additional false polygons left over from modelling, and inverted normal orientation) also appear in meshes reconstructed from scan data. In general, BRep models are not exact and only approximate the modelled geometry with limited precision, which is a serious issue when modeling highly valuable cultural heritage objects.

\section{Parameterization and operability}

The support of model generation with variable parameters is important when artifacts classification and authorship identification are involved. BRep has limited or no support for parameterization. When parameters are changed, BRep models have to be re-generated using a separate, high level procedure or method. A user may need to apply further specific operations on models such as offsets, blends and shape deformations and metamorphosis, which have limited or no support within BRep modeling systems.

\section{Manufacturability}

BRep models dramatically increase in size and become very hard or impossible for current hardware systems to visualize and even more difficult to cross-section as is required by many digital fabrication systems and processes. While current digital fabrication systems have limited resolutions, in recent years they have achieved ever greater accuracy and this trend is expected to continue. Even so, it is currently not uncommon to see defects or missing sections of an object, largely due to the complexity involved in creating proper cross-sections from the standard STL format, which represents the object surface by a set of disconnected triangles.

In contrast to the popular scanning and meshing techniques, we apply an approach utilizing real measurements, constructive modeling, and digital fabrication. The designer interactively creates constructive models of 3D objects with some degree of automation rather than using completely automatic mesh reconstruction from input data. The benefits of using such modeling techniques include a better understanding of the physical relationships of the components of the actual structure, as well as the processes used to create the original object. The created 3D models can replicate the actual construction of the original object itself, including features normally hidden to the eye, such as interior bracketing, and the model can be deconstructed to reveal such hidden features. This approach is especially valuable if the real object has been lost, destroyed or damaged. The goal is to create a $3 \mathrm{D}$ model of the object that is as complete as possible - to represent its internal structure, design logic (showing how components are interconnected or layered), and history of the shape construction, as well as time-dependent aspects and other parametric dependencies.

The Function Representation (FRep) [18, 19] was selected as the primary geometric model in our project. In FRep, a 3D object is represented by a continuous function of point coordinates as $F(x, y, z) \geq 0$. A point belongs to the object if the function is non-negative at the point. The function is zero on the entire surface (called usually an 
implicit surface) of the object and is negative at any point outside the object. The function can be easily parameterized to support modeling of a parametric family of objects. The HyperFun language [23, 24] was introduced for teaching and practical use of FRep modeling. The open and simple textual format of HyperFun, its clearly defined mathematical basis, its support of constructive, parameterized and multidimensional models, its support by free and open source modeling and visualization software, and its ease of use make it a good candidate as a tool for preservation and digital fabrication of cultural heritage objects. Additional discussion on shape representations for cultural heritage preservation can be found in [16].

The earlier stages of this project, namely modeling existing lacquer ware artifacts and Web presentations of the models were reported in $[16,25]$. In this paper we concentrate on the automation of the fabrication of traditional craft items as well as experimental modeling and manufacturing of new designs and forms. The test application areas for the project are traditional crafts from different cultural backgrounds, namely Japanese lacquer ware and Norwegian carving.

\section{LACQUER WARE MODELING AND FABRICATION}

Production of traditional Japanese lacquer ware called shikki utilizes manual techniques involving thin pieces of wood which are then assembled, painted in different colours, and covered by the natural lacquer called urushi. There are many different types of shikki items: boxes, small drawers, stands, bowls, sake cups and pots, spoons, chopsticks, notebooks, and even ball pens and pencils. These items are quite different in their topology, geometry, and texture. As with other traditional crafts, shikki is becoming a fading industry. The viability of cheap plastic production brings economic pressure to this craft industry, thus making the necessity of the craft preservation even more actual.

Modeling shapes of typical lacquer ware items and the presentation of them as virtual objects on the Web was implemented as follows. First, several 3D computer models of traditional Japanese lacquer ware items were created in HyperFun (see an example in Fig. 1). Then, polygonal approximations of object surfaces were made using the HyperFun Polygonizer software and the generated mesh was exported to the VRML (Virtual Reality Modeling Language) format. We scanned colour textures directly from lacquer ware objects with planar surfaces and from photographs. The obtained polygonal models have been textured using traditional tools like 3D Studio Max.

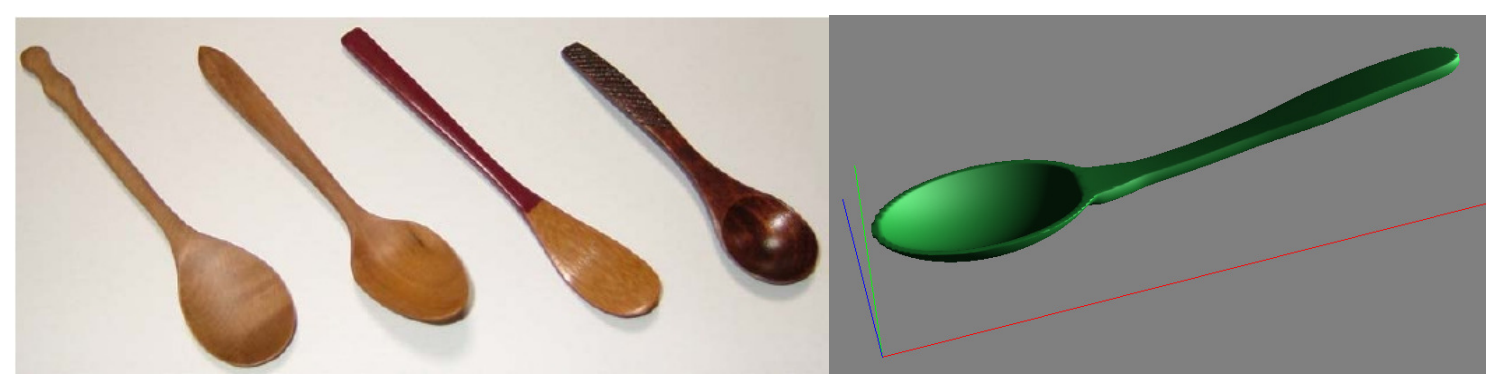

Figure 1: Japanese lacquer ware spoons (left) and a spoon modelled in HyperFun (right) 
Finally, the Web site Virtual Shikki [26] was created with the models of textured lacquer ware box, tray, cup, stand, sake pot, and a full sake set. A HyperFun model is available for each object at the Web site and each image is linked to the corresponding VRML model, which can be downloaded and visualized using any VRML viewer such as CosmoPlayer. The purpose of Virtual Shikki on the Web is to allow people to remotely appreciate the beauty of the object's shapes and textures. This is important both from cultural and commercial points of view.

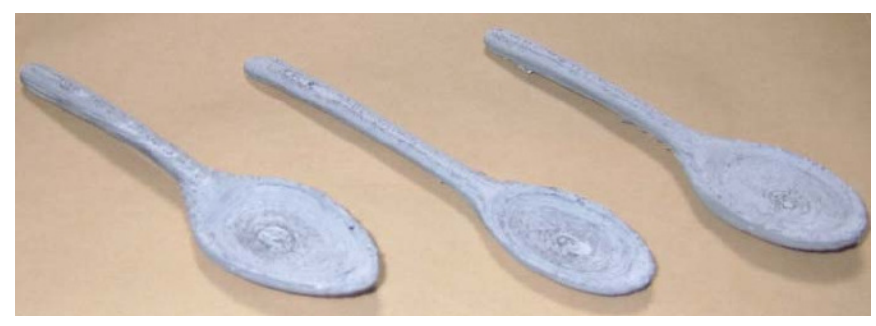

Figure 2: Spoon models fabricated by a rapid prototyping KIRA Solid Center machine using the paper laminating process.

We experimented with different digital fabrication equipment to produce the designed items. The objects fabricated by many rapid prototyping machines, can be used mainly for visual inspection purposes. The materials often used are too fragile and depending on the object, fine detail and finishing is not possible. For example, in Fig. 2 spoon models are shown which have been fabricated using a paper laminating KIRA Solid Center rapid prototyping machine.

We have primarily experimented with a low-cost and easy to use desktop milling machine (or a 3D plotter) Modela MDX-20 (Roland DG, Japan). In contrast to 3D printers, the Roland Modela supports even hard woods as the material, and is able to produce fine detail and design. The spoon model shown in Fig. 1 was produced out of wood using a Modela MDX-20 and is shown in Fig. 3.
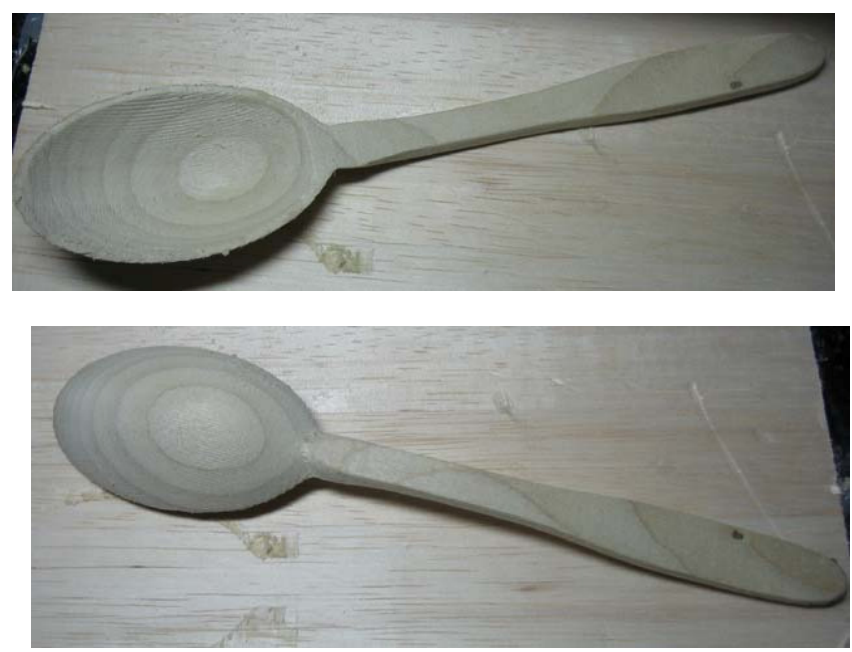

Figure 3: A spoon model fabricated using a Modela machine. 

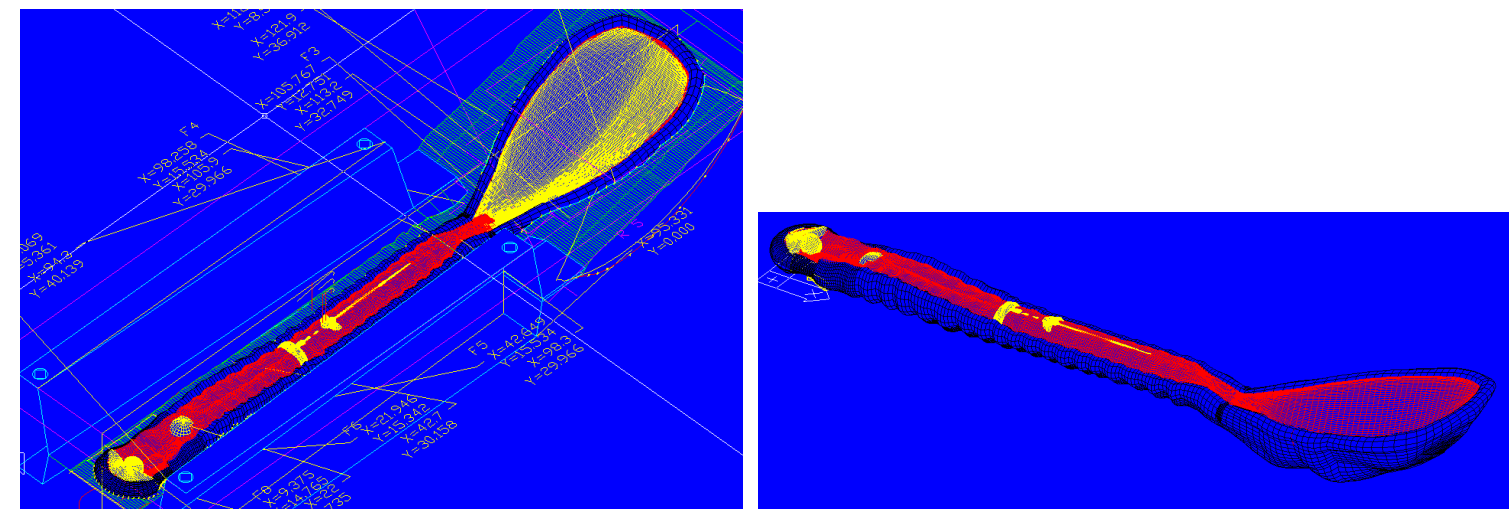

Figure 4: New 'organic' spoon design.

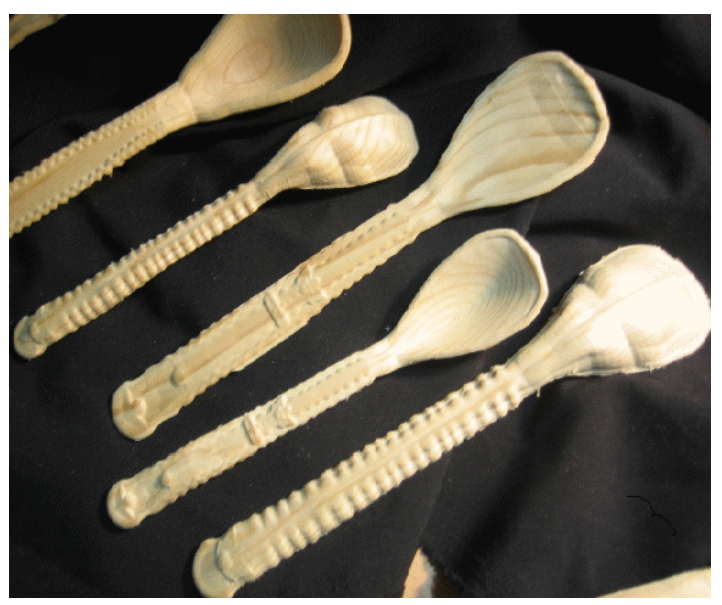

Figure 5: New spoon designs fabricated of wood using a Modela machine.

In addition to modeling and fabrication of traditional lacquer ware items in the project, we are trying to expand the variety of designed objects. New designs of spoons and bonbonnieres were proposed (Figs. 4 and 6) and an experimental fabrication technique was tested (Figs. 5 and 7). An important part of the fabrication process is the tooling. Such tooling can be seen in Fig. 7 that the top and the bottom of the bonbonniere lid were fabricated using wax to hold the piece in place during milling.

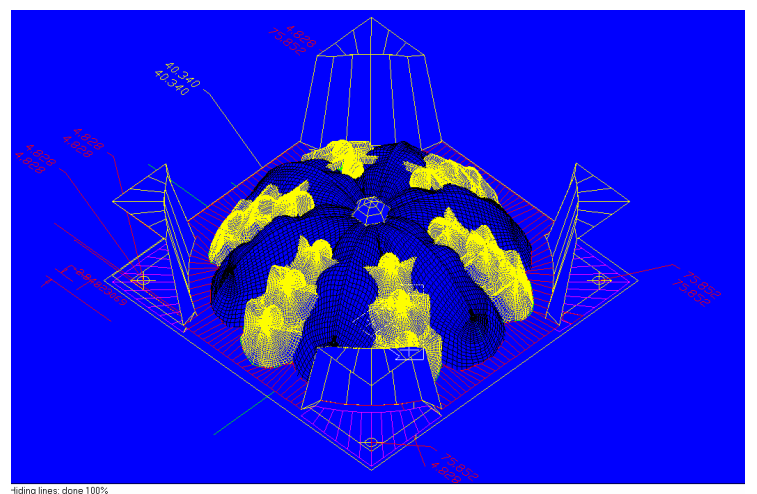

Figure 6: New bonbonniere design. 

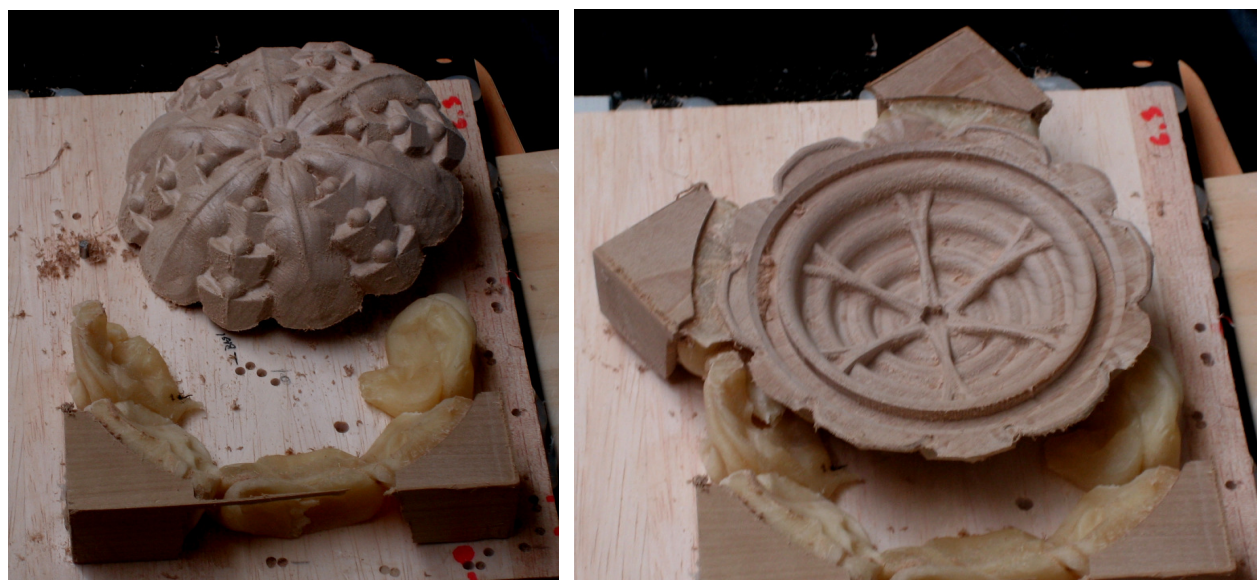

Figure 7: A fabricated wooden bonbonniere lid with tooling for a Modela machine.

\section{MODELING AND FABRICATION OF NORWEGIAN CARVING}

The initial craft artifact for this experimental case study was an original wood carving from the Lyngen region of Norway (Fig. 8). This image is considered a symbol of the local community and there was an idea to create its $3 \mathrm{D}$ relief version and to use for producing jewellery and other purposes.

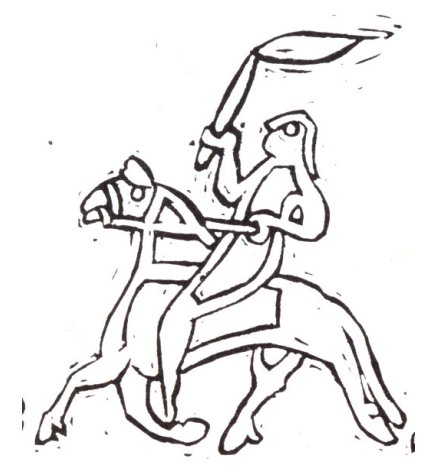

Figure 8: An original wood carving from the Lyngen region of Norway.

The implementation process included measurements of the original carving image, modeling the object using HyperFun, and producing a mold for casting. The 3D model shown in Fig. 9 is made as a composition of relief models of several parts of the carving such that it is suitable for casting. The relief models were generated using the polygon-to-function conversion procedure described in [15] and applied to the outlines of the elements of the original carving. The cast form (Fig. 10 left) was fabricated out of modeling wax on a Modela MDX-20. The total milling time for a surface finished object of the model was 18 hours. This wax model was then used to make a mold, a negative cast using gypsum material (Fig. 10 right), such that it can be used as a medium for casting. 

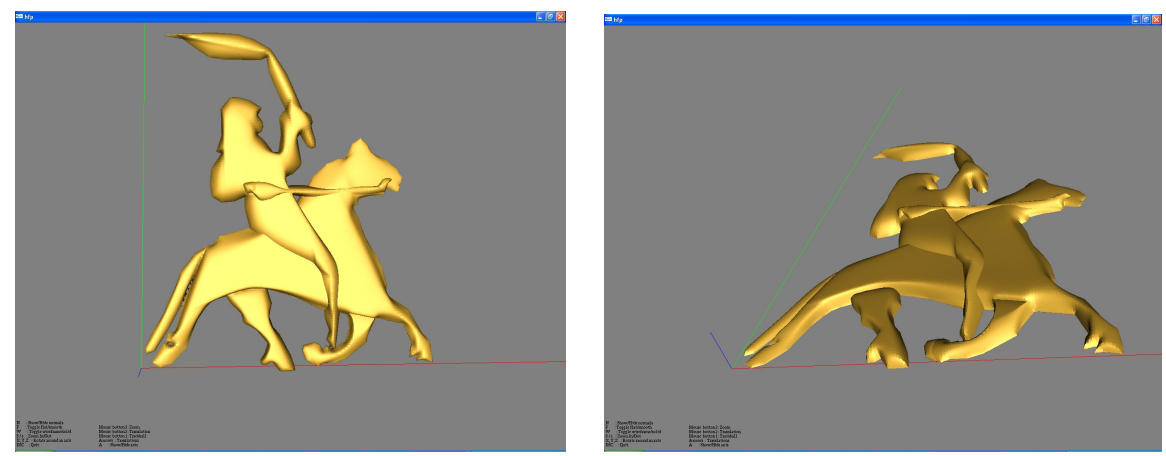

Figure 9: 3D HyperFun model of the original carving.
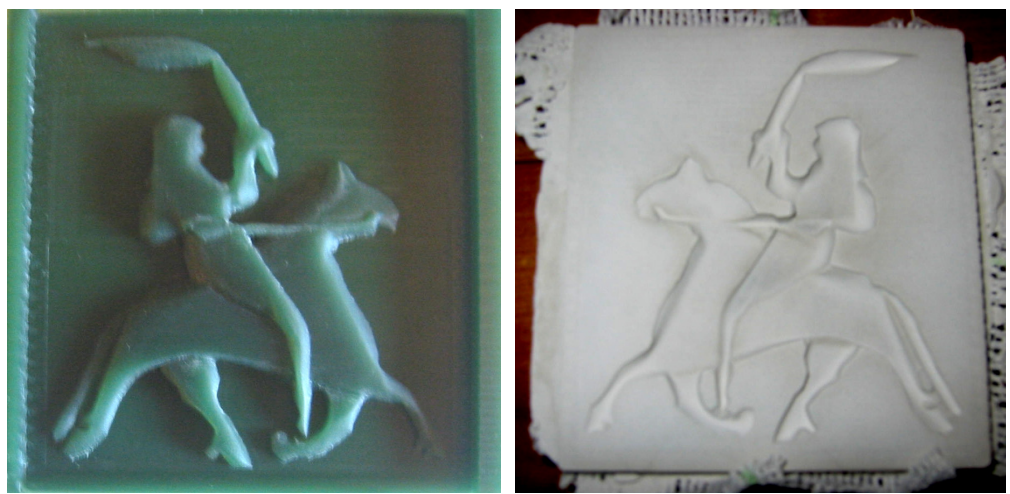

Figure 10: A wax model fabricated with a Modela machine (left) and

a negative gypsum cast form made using the wax model (right).

The process of creating a physical representation of a HyperFun model using the Roland Modela MDX-20 is a careful and time-consuming process. The process starts by creating a STL file from the HyperFun model. This file is imported into Roland DG's Modela Player milling software. Then the STL model is scaled to fit the limitations of the MDX-20's bed and the size of the object desired. The material must also be cut to the right size, just slightly larger than the object to be milled. When using the MDX-20, in general there are three phases of the milling process for every side that is milled. First the material that will be milled must be planed into a flat surface, parallel to the machine's bed. Then a rough milling is made, removing most of the material and leaving a rough layer above the final surface that yields an approximation of the object's shape. Finally, a finishing milling pass is made removing the rest of the material and providing a finished surface and object. In practice it may be necessary to also spot mill specific areas of the object for more detail, so that you may have not just one but many finished milling processes. It is normal that for each step of the milling process, a different size bit is used, usually from large to small. For each one of these steps, bit sizes, milling style and milling speed must be selected and milling paths using these settings are then generated by Roland's software. The material is then affixed to the milling bed (for a one-sided mill) using hot glue at the edges of the material to be milled, locking it in place. Each milling process is run in order from the plaining mill to the last finishing mill, producing a finer surface each time. Between most mills the waste must be collected, the bit is changed to a finer one and machine must be recalibrate to the surface. It should also be noted, that there is some loss of surface detail and aliasing that can be observed in the final models when generating milling paths from the STL format rather than from 
HyperFun directly.

While the resulting molds could be taken to a professional jeweller, we also experimented with a clay developed by Art Clay, to produce jewellery ourselves using these molds. The clay is composed of 1-20 micron sized silver particles, organic binders, and water. During the firing process, the organic binders burn away, which allows the silver particles in the clay to become denser and stronger resulting in an object which is $99.9 \%$ silver. After some experimentation with the different forms of this material, we found the best results for filling the mold came from using the syringe type clay. Following the instructions from Art Clay, the filled mold was heated in an oven for a short period to dry the clay. After the silver object was removed from the mold and it was fired. Both a professional kiln was used, as was an inexpensive portable kiln, each with similarly successful results. After polishing the object what resulted was a professional quality silver object. (Fig. 11).

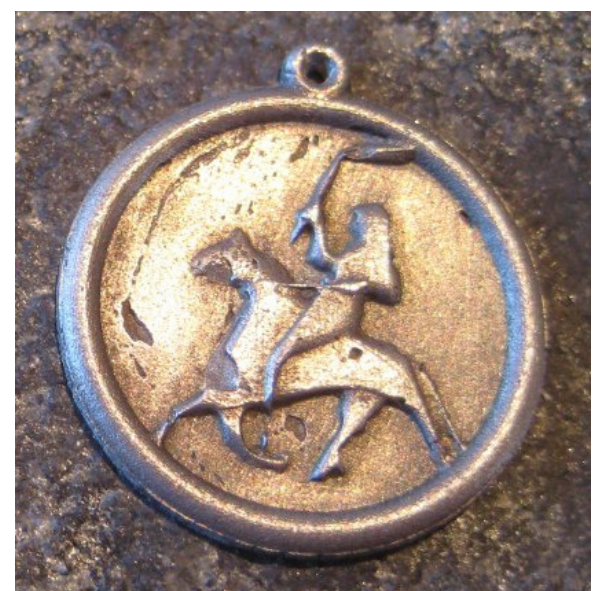

Figure 11: A finished piece of silver of the Norwegian rider carving.

This process was further developed into a curriculum to teach high school design students how to design and fabricate their own creations. The students were taken through the entire process, starting with the design conception on a piece of paper (Figure 12 left). After a few sessions in 3D software and HyperFun in particular, they transferred their designs from paper to digital 3D models. Using the process described above, a mold was made for each students' design using a Roland MDX-20. Next the students used the Art Clay material to fill their molds, which were then dried, fired, polished and further stylized by the students using traditional jeweller's tools. Each of the students who participated in the class ended up with a digital model and a real piece of silver jewellery from their original paper design (Figure 12 right). 

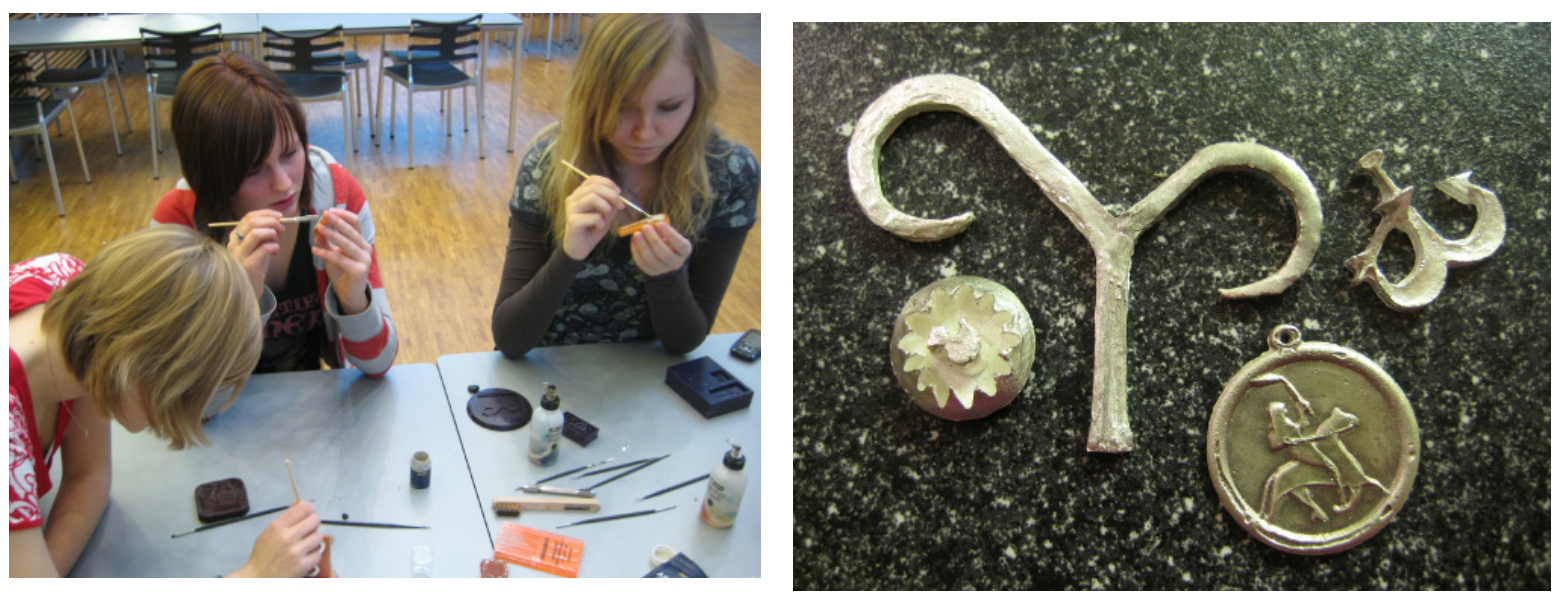

Figure 12: Some of the students from the design school Breivang in Troms $\phi$, Norway, filling their molds with silver clay (left) and a few of the finished silver objects (right).

\section{SYSTEM EVALUATION AND DISCUSSION}

Until recently, there were several problematic issues in using FRep in cultural heritage applications: slow rendering, lack of support for interactive modeling, Web presentation and digital fabrication. We have dealt with these issues while developing a usable modeling, rendering and fabrication system as described below.

\section{Real-time direct rendering}

For some time, the main disadvantage of applied FRep modeling was time-consuming function evaluation at the given point and therefore slow object rendering, which was retarding interactive applications. Typically, an FRep object had to be approximated by a polygonal mesh, which ten could be rendered using graphics hardware. Recently, a method was introduced for direct ray-casting and ray-tracing FRep objects employing Revised Affine Arithmetic and its extensions [27]. When implemented on the GPU, this method provides real time direct rendering (4 frames per second) of the entire sake set from the Virtual Shikki project [26]. 


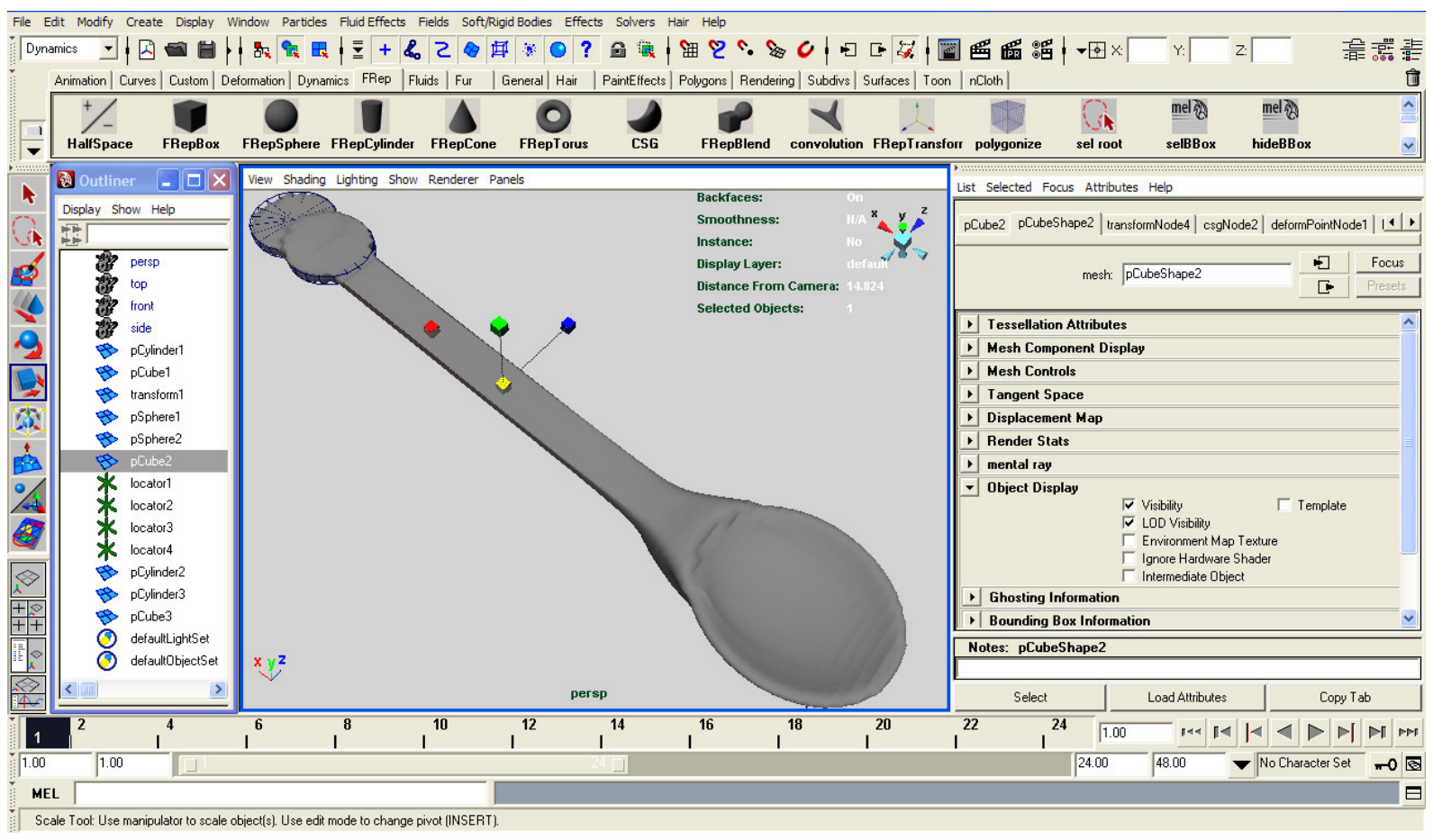

Figure 13: Interactive FRep modeller as a plug-in to Autodesk Maya.

\section{Interactive modeling}

The fast direct rendering provided a basis for the implementation of interactive modeling system thus avoiding tedious coding in HyperFun. Our prototype interactive modelers have been implemented as plug-ins to Autodesk 3ds Max and Maya systems. Different modes of operations are provided for the user such as direct objects manipulation, command line and tree interface (see Fig. 13). For certain applications a difficulty of constructive modeling is that it usually is not an automated process, taking time and requiring some skill in $3 \mathrm{D}$ modeling However, it can be beneficial and even critical to have a constructive model for advantages and benefits discussed earlier. Therefore, we examine in $[16,17]$ several approaches to the modeling automation such as fitting a parameterized FRep template model to a cloud of data points, and extraction of the logical structure of the model in the form of the construction tree using genetic algorithms.

\section{Web presentation}

The choice to use the VRML format for the Web presentation of 3D virtual objects was a natural choice at the earlier stages of our project [25], as until recently it was the standard format for representing 3D models on the Web. However, VRML has well-known drawbacks such as huge data files and therefore long downloading time. For example, an average size VRML file is 100-500 Kb. Therefore more concise Web3D formats should be considered in the future, and HyperFun is one such format. In comparison to VRML with the size of the full sake set HyperFun file is $4.5 \mathrm{Mb}$, the HyperFun models for all lacquer ware items do not exceed $5 \mathrm{~Kb}$. In this sense, we can conclude that because of the intrinsic nature of HyperFun to reduce everything to a mathematical function which provides a high level of compression, it should be considered as a lightweight network protocol. A radical solution to use HyperFun for the Web presentation is a plug-in to a Web browser [28]. This plug-in allows for the transfer of the small HyperFun models and could unfold a polygonal mesh or support direct 
rendering suitable for interactive visualization.

\section{Direct fabrication}

Several of the FRep models in this paper have been manufactured using a desktop fabrication machine with a standard input in the form of the STL file. These STL files were imported into the standard software package for driving the machine where they were checked for surface defects and then machine paths were generated based on layered slices of the STL and manufacturing constraints. During the manufacturing process, the STL file format created many issues for the slicing, path planning and fabrication of some of the fine features of the models. A much better approach is to directly fabricate the FRep model without poor intermediate formats such as STL. This has been previously proposed in [29] and is an active ongoing focus of our research. One possibility is to produce a raster image for each layer of manufacturing at the machine resolution, which is an acceptable input for some existing machines. Thus the FRep model could be procedurally defined on a voxelgrid that corresponds directly to the layer thickness and to the pixel spacing of the printing process. Another choice is to directly control the digital fabrication process including the tool motions and material deposition. However, the obstacles presented by the proprietary nature of most digital fabrication technologies, such as access to machine protocols and control commands, require open hardware systems such as the Fab@Home or RepRap 3D printers. Unfortunately, the resolution of these free and open source systems is currently not competitive with high end commercial machines necessary for fine resolution microstructures. Another direction of future work is the development and experimentation with multi-material 3D printers to reproduce surface painting and other features of craft items.

Our experience shows that inexpensive desktop fabrication equipment with appropriate software can give ground for the preservation and support of existing craftsman techniques as well as for new approaches to design and fabrication. We have shown that digital technology can be used not to disrupt or replace traditional processes, but extend and enhance them. This means that traditional shapes and forms can not only be preserved, but continue to develop.

Our project is oriented to the living preservation of traditional craft techniques by providing a platform to both enhance traditional crafts using digital technology and computationally capture key information about the shapes, structures, and to some degree the processes used in traditional crafts. We propose the creation of new digital and physical artifacts that reference and extend these traditional processes, and demonstrate how it can be applied in education for attracting young people to traditional crafts, computing and fabrication. As the number of masters of traditional crafts is substantially decreasing, we hope such research can help to revitalize and provide for the living preservation of cultural heritage.

\section{REFERENCES}

[1] A. Addison. 2000. Emerging trends in virtual heritage, IEEE Multimedia, Special Issue on Virtual Heritage, vol. 7, No. 2, $22-25$.

[2] D. Arnold. 2008. Pasteur's Quadrant: cultural heritage as inspiration for basic research in computer science, ACM Journal on Computing and Cultural Heritage, vol. 1, No. 1, Article 1. 
[3] Chee Kai Chua, R. Gay, W. Hoheisel. 1997. Computer aided decoration of ceramic tableware, Computers and Graphics, vol. 21, No. 5, 641-653.

[4] D. Tsiafakis, A. Tsompanopoulos, G. Pavlidis, D. Papadopoulou, N. Tsirliganis, V. Evangelidis, C. Chamzas. 2003. Archiving cultural objects in the 21st century: pottery from Karabournaki, 16th International Congress of Classical Archaeology AIAC 2003, Harvard University Museums, Boston/Cambridge, USA.

[5] R. Shaughnessy. 1999. An approach to the digitization of the collection at the Museum for Textiles, Museum for Textiles, Toronto, Canada.

[6] M. Levoy, K. Pulli, B. Curless, S. Rusinkiewicz, D. Koller, L. Pereira, M. Ginzton, S. Anderson, J. Davis, J. Ginsberg, J. Shade, D. Fulk. 2000. The Digital Michelangelo Project: 3D scanning of large statues. Siggraph 2000, Computer Graphics Proceedings, ACM Press, 131-144.

[7] S. Havemann, D. Fellner. 2004. Generative parametric design of Gothic window tracery. Proceedings of the $5^{\text {th }}$ International Symposium on Virtual Reality, Archeology, and Cultural Heritage. 193-201.

[8] J. Rossignac (Ed.). 1994. Special issue on interactive sculpting, ACM Transactions on Graphics, vol. 13, no. 2.

[9] ZBrush Overview, http://www.pixologic.com/zbrush/

[10] S. Mizuno, M. Okada, J. Toriwaki. 1998. Virtual sculpting and virtual woodcut printing, The Visual Computer, vol. 14, No. 2, 39-51.

[11] S. Mizuno, D. Kobayashi, M. Okada, J. Toriwaki, S. Yamamoto. 2003. Virtual sculpting with a pressure sensitive pen, ACM SIGGRAPH 2003 Sketches \& Applications.

[12] A. Sourin. 2001. Functionally based virtual embossing, The Visual Computer, vol. 17, No. 4, 258-271.

[13] Levinski K, Sourin A. 2007. Interactive function-based shape modelling, Computers \& Graphics, vol. 31, No.1, 66-76.

[14] S. Mizuno, T. Kasaura, S. Yamamoto, M. Okada, J. Toriwaki. 2000. Automatic generation of woodblocks for virtual printing, 15th International Conference on Pattern Recognition (ICPR'O0), vol. 4, 44-59.

[15] A. Pasko, V. Savchenko, A. Sourin. 2001. Synthetic carving using implicit surface primitives, Computer-Aided Design, Elsevier, vol. 33, No. 5, 379-388.

[16] C. W. Vilbrandt, G. I. Pasko, A. A. Pasko, P.-A. Fayolle, T. Vilbrandt, J. R. Goodwin, J. M. Goodwin, T. L. Kunii. 2004. Cultural heritage preservation using constructive shape modeling, Computer Graphics Forum, vol. 23, No.1, 25-41.

[17] P.-A. Fayolle, A. Pasko, E. Kartasheva, C. Rosenberger, C. Toinard. 2008. Automation of the volumetric models construction, in Heterogeneous Objects Modelling and Applications, Lecture Notes in Computer Science, vol. 4889, Springer Verlag, 214-238.

[18] A. Pasko, V. Adzhiev, A. Sourin, V. Savchenko. 1995. Function representation in geometric modeling: concepts, implementation and applications, The Visual Computer, vol.11, No. 8, 429-446.

[19] A. Pasko, V. Adzhiev. 2004. 'Function-based shape modeling: mathematical framework and specialized language', Automated Deduction in Geometry, Lecture Notes in Artificial Intelligence 2930, Ed. F. Winkler, Springer-Verlag, Berlin Heidelberg, 132-160.

[20] M. Balzani, M. Callieri, M. Fabbri, A. Fasano, C. Montani, P. Pingi, N. Santopuoli, R. Scopigno, F. Uccelli, A. Varone. 2004. Digital representation and multimodal presentation of archeological graffiti at Pompei, Proceedings of 5th International Symposium on Virtual Reality, Archaeology and Intelligent Cultural Heritage VAST 2004, Eurographics Association, 93-104.

[21] T. Allard, M. Sitchon, R. Sawatzky and R. Hoppa. 2005. Use of hand-held laser scanning and 3D printing for creation of a museum exhibit, Proceedings of 6th International Symposium on Virtual Reality, Archaeology and Cultural Heritage VAST 2005: Short and Project Papers, 97-101.

[22] M. Cooper, A. La Pensée, J. Parsons. 2006. The use of laser scanning and rapid manufacturing techniques for museum exhibitions. Proceedings of 7th International Symposium on Virtual Reality, Archaeology and Cultural Heritage, EPOCH Publication, 65-71.

[23] V. Adzhiev, R. Cartwright, E. Fausett , A. Ossipov , A. Pasko, V. Savchenko. 1999. HyperFun project: a framework for collaborative multidimensional F-rep modeling, Implicit Surfaces '99, Eurographics/ACM SIGGRAPH Workshop, J. Hughes and C. Schlick (Eds.), 59-69. 
[24] R. Cartwright, V. Adzhiev, A. Pasko, Y. Goto, T. Kunii. 2005. Web-based shape modeling with HyperFun, IEEE Computer Graphics and Applications, vol. 25, No. 2, 60-69.

[25] G. Pasko, A. Pasko, C. Vilbrandt, T. Ikedo. 2001. Virtual Shikki and Sazaedo: Shape modeling in digital preservation of Japanese lacquer ware and temples, Spring Conference on Computer Graphics, R. Durikovic, S. Czanner (Eds.), IEEE Computer Society, 147-154.

[26] Virtual Shikki Web site, http://hyperfun.org/wiki/doku.php?id=apps:shikki

[27] O. Fryazinov, A. Pasko, P. Comninos. 2010. Extending Revised Affine Arithmetic for fast reliable ray-tracing of procedurally defined implicit surfaces, Proceedings of International Conference on Computer Graphics Theory and Applications GRAPP 2010, 199-207.

[28] T. Vilbrandt, O. Fryazinov, C. Stamm, A. Pasko. 2008. A Web oriented function-based volume modeling framework, Technical Report TR-NCCA-2008-02, ISBN 1-85899-123-4, The National Centre for Computer Animation, Bournemouth University, UK.

[29] T. Vilbrandt, E. Malone, H. Lipson, A. Pasko. 2008. Universal desktop fabrication, in Heterogeneous Objects Modelling and Applications, Lecture Notes in Computer Science, vol. 4889, Springer Verlag, 259-284. 